\title{
Third Eye -An Efficient Home Security Automation System
}

\author{
Harikrishnan G.R. \\ Asst. Professor \\ MESCE \\ Kuttipuram India
}

\author{
Noufal V.P. \\ Student \\ Veshnam Para (H) Edayur Post \\ India
}

\author{
Latheesh S. \\ Student \\ Vilakkithala $(\mathrm{H})$ \\ Porkulam post \\ India
}

\author{
Shakkir Ummer U. \\ Student \\ Uliyath $(\mathrm{H})$ \\ Chamravattam Post India
}

\author{
Nithin S. \\ Student \\ Kodakkad $(\mathrm{H})$ Kodakkad Post \\ India
}

\begin{abstract}
Home automation is becoming more and more popular day by day due to its numerous advantages. This paper aims at designing a home security automation using Raspberry Pi. Home automation is an emerging field in information technology. Nowadays security cameras (CCTV) are available for recording different events happening in home and offices. These cameras are used only for recording videos and these recorded video can later be used for analysis. This paper introduce a system that constantly monitors the house perimeter and notify the owner of the house when there is a visitor or intruder. The owner is informed with an image of visitor and time of visiting. The system is implemented using Raspberry Pi. The system is capable of notifying the user by Email, SMS, Call and Whatsapp instantly using python scripting
\end{abstract}

\section{General Terms}

Security, Automation

\section{Keywords}

Home Security Automation, CCTV, Raspberry Pi, Email, SMS, Python.

\section{INTRODUCTION}

Today, the use of home automation is increasing day by day. Home automation an emerging field in information technology. Home automation is simply controlling different activities of home with the advanced technologies. Like controlling electronic devices, switching on/off electrical appliances with cellular devices from a different location. It allows the user to perform different action of home from a remote place

Home security automation is s field of home automation. It concentrate on the security aspects of homes and offices. It enables remote surveillance of homes and offices to improve the security of home and offices using information technology and wireless communication. The popularity of home security automation has been increased in greatly in recent years due to much higher affordability and simplicity. This can be achieved by local networking or by remote control. Security systems are valuable tools in protection against many of the dangers that can happen in and around a home. These systems safeguard homes from intruders and burglars. Home security begins with home safety. Home safety begins with homeowners taking steps to protect their home and its residents. One of the best ways for homeowners to have a safe home is to install a security camera system

Raspberry $\mathrm{Pi}[1]$ is a credit card-sized single board microcomputer developed in UK by the Raspberry $\mathrm{Pi}$ Foundation. It was finally released in February 2012. It has provided new opportunities to enhance tools for education. It also helps to enable developers to access in affordable cost and easy to program. It primarily uses Linux kernel based operating system, the latest version of Pi support windows 10 operating system. The ARM11[1] is based on version 6 of the ARM on which several computer versions of Linux no longer run including Ubuntu. They are available in three different models, A, B and $\mathrm{B}+$. Figure 1 shows a Raspberry Pi model B. Main objective of this paper is to implement home security automation using Raspberry Pi. This is achieved through installing a camera. By implementing some complex algorithms the system should be able to capture picture when a visitor or intruder enter into the house perimeter

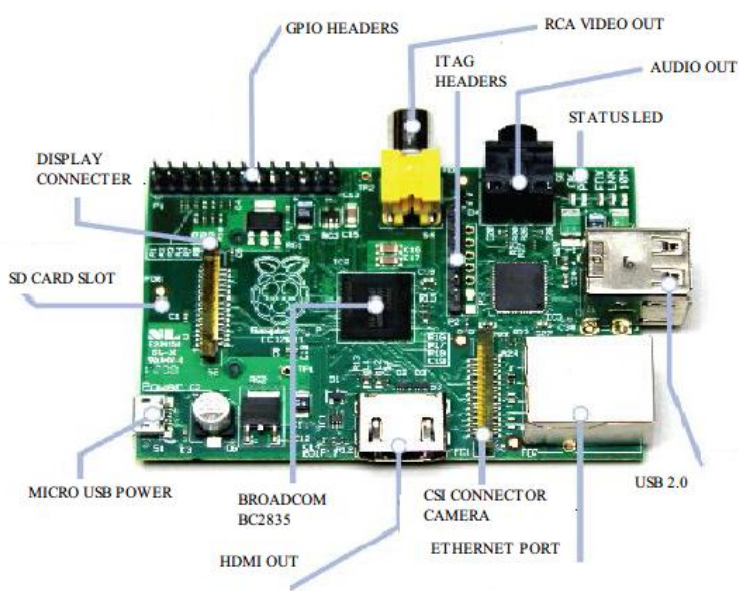

Figure 1 Raspberry Pi model B .

\section{LITERATURE SURVEY}

\subsection{Closed Circuit Television}

Closed circuit televisions are also known as CCTV. These systems are the most commonly used security system. CCTV cameras record live videos or capture images. Two kind of 
CCTV available are wireless and wired. These systems are capable of recording live videos for a time period. CCTV cameras can be applied anywhere but it is most commonly used for security purposes in banks, airports, shoping centers etc. CCTV cameras continously monitors a particular place or sometimes operated for a particular event. The earliest CCTV systems were not capable of recording and storing information. It was the development of reel-to-reel media which became crucial turning point in the enhancement of modern systems. These systems are highly influential in crime prevention, industrial processes, traffic monitoring etc. Digital Multiplexing allows several CCTV cameras to record at once. These enables the recording events at various locations. The latest CCTV systems enhanced its performance by coupling with Internet. Industrial processes that take place under conditions dangerous for humans are today often monitored with CCTV systems. Chemical Industry and Nuclear Industry are the examples of industries in which these systems superwise and provide a highly beneficial service.

\subsection{Home Alarm}

Security alarm[4] is a system designed to detect intrusion and unauthorized entry into a building or area. These systems are used in residential, commercial, Industrial and military properties for protection against burglary or property damage, as well as personal protection against intruders. Some alarm system serves a single purpose burglary protection; the combination system provide both fire and intrusion protection. Systems range from small self-contained noisemakers, to com plicate d, multi-area systems with computer monitoring and control. Alarms comes with different of sensors such as Passive Infrared Detectors (PIR), Ultra Sonic Detectors, Microwave detectors, photoelectric beams, glass-break detection, vibration or inertia detectors and so on.

\subsection{Raspberry pi}

A Raspberry Pi is a credit card sized computer board. Like a PC, it has RAM, Hard Drive (SD Card), Audio and Video ports, USB port, HDMI port, and Ethernet port. With the Pi, users can create spread sheets, word-processing, browse the internet, play high definition video and much more. It was designed to be a cost friendly computer for users who needed one. There are three models, Model A, B and B+. Model B+ is the faster containing $512 \mathrm{MB}$ of RAM as well as the ability to over clock. The Raspberry Pi gets powered via a regular $5 \mathrm{~V}$ micro USB port and uses between $700-1000 \mathrm{~mA}$. For projects requiring more power than $1 \mathrm{Amp}$ an externally powered USB hub is needed. The GPIO pins on the board require $16 \mathrm{~mA}$ each, the camera module requires $250 \mathrm{~mA}$, HDMI requires $50 \mathrm{~mA}$ while keyboard and mice requirements vary. Powering the Raspberry Pi using batteries is possible however it is not recommended because batteries do not provide stable power which can harm the system. As mentioned above the Raspberry Pi's hard drive is the SD card inserted into it. The minimum requirement for the SD card is $4 \mathrm{~GB}$ and it can go up to $32 \mathrm{~GB}$.

Raspberry Pi is Linux based and the OS is stored on the SD card. There are several distributions that can be installed and are specifically created for the Raspberry Pi. There is an OS named NOOBs specifically for individuals who are new to the Pi. Other OS's include Raspbian[5], Pidora, RaspBMC, ARC and OpenELEC. In this system we are using the Raspbian operating system. In order to install the OS onto the SD card the raw image has to be first downloaded from the Pi's website. Once it is downloaded, the SD card has to be formatted using the software SDFormatter. Once it is formatted, the software Win32DiskImager has to be used to write the image onto the SD card. The SD card can then be inserted into the Raspberry Pi and booted up using an LCD display and a keyboard. The power source, SD card and keyboard are necessary components for setting up the Raspberry Pi.

The Pi does not contain its own Wi-Fi connection yet it does have an Ethernet port. It can also support certain models of Wi-Fi dongles such as the Edimax Nano Adapter. Plugging in an Ethernet cable to a router will automatically enable the internet, however, with a Wi-Fi dongle there are various steps that are need to be followed to properly make it function. With these methods, the browser can be used and also access to the Internet is needed to install various packages through the terminal. The default programming language provided by Raspberry Pi is Python. Other languages such as Java, C, and $\mathrm{C}++$ can also be installed and used if the programs are installed on the system. It is important to note that installing programs that use these languages might take up a large amount of space on the SD card. This can also cause the system to slow down. Over clocking is possible, however, it is important to note that the proper methods have to be followed or else this may harm the Pi. Table 1 shows the comparison between raspberry pi models $\mathrm{A}$ and $\mathrm{B}$

\begin{tabular}{|c|c|c|}
\hline Specifications & Model A & Model B \\
\hline Processor & $\begin{array}{c}\text { Broadcom BC2835 } \\
\text { (CPU GPU) }\end{array}$ & $\begin{array}{c}\text { Broadcom BC2835 } \\
\text { (CPU GPU) }\end{array}$ \\
\hline Memory & 256MB SD RAM & 512MB SD RAM \\
\hline USB Ports & 1 USB 2.0 Ports & 2 USB 2.0 Ports \\
\hline Networking & None & Ethernet Port \\
\hline Video Out & HDMI & HDMI \\
\hline Audio Out & Audio & Audio \\
\hline Secondary Storage & SD Card Slot & SD Card Slot \\
\hline Power Supply & Micro USB for power & \\
\hline
\end{tabular}

Table 1 Comparison of Raspberry Pi model A and B

\subsection{Camera and Motion Detection}

The Camera Board[6] on the Raspberry Pi is a small printed circuit board. The PCB is connected to a ribbon cable which connects to the Pi itself on its own port. The ribbon can be extendable. The camera on the board is very small (5MP camera); and is comparable to the ones used on cell phones today. As for now it is the only Camera made specifically for the Pi therefore these specifications cannot be updated. Since it uses $250 \mathrm{~mA}$, externally powering the Pi should be sufficient enough for the camera. If more items are connected, however, once again an externally powered USB hub is needed. A USB powered webcam can be used to be able to achieve motion detection. For this case only a camera board is needed. In 
order for the camera to be enabled it is necessary to go to the raspberry pi configuration settings and selecting Enable Camera. The camera can be used as a regular camera as well as a motion detecting camera.

\section{SYSTEM MODEL}

\subsection{Proposed System}

The proposed system consist of a camera attached to a Raspberry Pi board that constantly monitors the perimeter of the house. By using motion sensors and motion detection[7] algorithms, the system detects the presence of a human being. The camera capture the visitor and sends the picture along with visiting time to house owner through an e-mail and SMS. Thus the house owner gets instant notification and can take action.

Some advantages of the proposed system are

- Instant Notification

- Wireless System

- Secure

- Easy Implementation and maintenance

- Cheaper to implement

- Software updating is possible

- Works with basic phones

\subsection{SYSTEM ARCHITECTURE}

The architecture of Home Security Automation consists of a camera module which captures images, sensing unit which can be either hardware or software. Figure 2 shows the architecture of the entire system. The architecture consist of an admin, and a user. The admin register the email address and mobile number of the user into the system. The data are stored into the database. Once a visitor enter into the house perimeter, the camera detects motion and captures the image of visitor. This image is then send to the registered email address of the user. The system is also capable of notifying the user through SMS, Call and even Whatsapp.

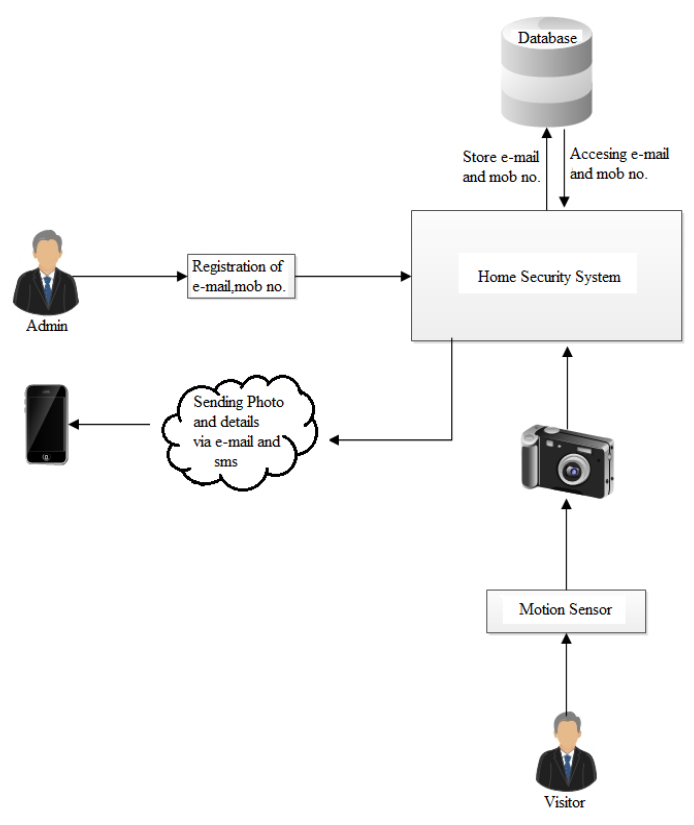

Figure 2-Architecture

\section{SYSTEM IMPLEMENTATION}

\subsection{Motion Detection}

Motion detect is the process of detecting the change in the surroundings relative to an object or a change in position of an object relative to its surroundings. Motion detector is a device can detects moving target, especially people. Motion detectors are widely used in lift, supermarket. Any place that most of the time no activity can use motion detector replace normal surveillance cameras. A motion detector is often completed as an element of a system that automatically executes alerts a user motion detected in an area. Motion detectors form an important component of energy efficiency, home control, automated lighting control, security, and other useful system In Raspberry pi Motion detection can be done using the software package called motion. By adjusting the threshold value and other significant parameters into a proper value, the system will be able to detect motion in a frame. Figure 3 illustrate the basic idea of motion detection.
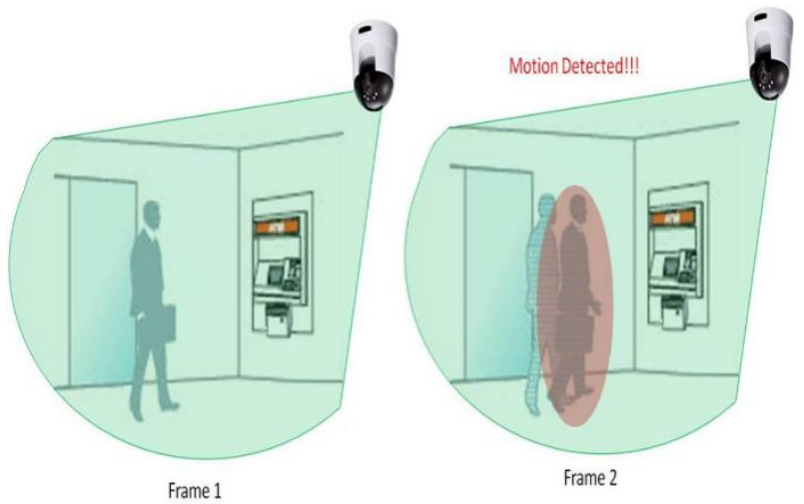

Figure 3 Motion Detection

\subsection{SSMTP and MPACK}

SSMTP[8] is a program which delivers email from a local computer to a configured mailhost (mailhub). It is not a mail server (like feature-rich mail server sendmail) and does not receive mail, expand aliases or manage a queue. One of its primary uses is for forwarding automated email (like system alerts) off your machine and to an external email address.

The mpack program encodes the named file in one or more MIME messages. The resulting messages are mailed to one or more recipients, written to a named file or set of files, or posted to a set of newsgroups.

SSMTP is used to configuring the email address in the system and Mpack is used to send email with attachment to the configured email address.

\subsection{Twilio}

Twilio[9] is a cloud communications (IaaS) company based in San Francisco, California. Twilio allows software developers to programmatically make and receive phone calls and send and receive text messages using its web service APIs. Twilio's services are accessed over HTTP and are billed based on usage. Twilio is used to facilitate SMS and Phone call.

\subsection{Yowsup}

Yowsup[10] is a python library that enables you build application which use Whatsapp service. Yowsup has been 
used to create an unofficial WhatsApp client. 'yowsup-cli' is a command Yowsup comes with a command line client exposes many yowsup capabilities for quick access in command line. At the moment it supports only a smaller set of yowsup function as it's still in under heavy development, but eventually it will expose every single yowsup capabilities.

\subsection{Performance Analysis}

CCTVs are only capable of recording videos and can only be used for future analysis mainly. Live CCTV systems are highly complex. The value of CCTV information has increased, resulting in a higher risk from hackers. Hacking CCTV footage has led to privacy issues. It is not possible to completely protect public security system from hackers. As the system connects to a network, hackers can hack into the system virtually from outside locations. Wired CCTV system has the disadvantage of being fixed to a particular area, meaning that the camera can't simply change the location. The installation and cabling of these camera is a difficult task that demands the assistance of professionals. As the CCTV system is constantly recording video footage from cameras it requires a high amount of storage space. The requirement of professionals and large storage space increase the installation and maintenance cost. Home alarms are not at all difficult to professional burglars. There is a possibility that the user can forget to enable the alarm when leaving. Almost all home alarm system have to shut the windows and doors properly before it is enabled. A major complaint about alarms is that most of the professional burglars are familiar with alarms and know precisely to deal with it. This system cannot capture image or video, so the identity of burglar is unknown if he escape.

\begin{tabular}{|c|c|c|c|}
\hline & CCTV & $\begin{array}{l}\text { Home } \\
\text { Alarm } \\
\end{array}$ & Raspberry Pi Camera \\
\hline $\begin{array}{c}\text { Video and } \\
\text { photo capturing }\end{array}$ & Yes & No & Yes \\
\hline $\begin{array}{c}\begin{array}{c}\text { Instant } \\
\text { notification to } \\
\text { user }\end{array} \\
\end{array}$ & No & No & Yes \\
\hline $\begin{array}{c}\text { Software } \\
\text { update }\end{array}$ & No & No & Yes \\
\hline $\begin{array}{c}\text { Additional } \\
\text { Camera } \\
\text { support } \\
\end{array}$ & Yes & NA & Yes \\
\hline Memory usage & High & NA & Low \\
\hline Cost & $\begin{array}{c}\text { Rs. } 10000- \\
30000 \\
\end{array}$ & $\begin{array}{l}\text { Rs. } 10000 \\
\text { Above }\end{array}$ & Rs. 4000 \\
\hline
\end{tabular}

Table 2 Comparison between existing and proposed system

From the comparison table it is clear that the existing systems are not capable of instant notification. The existing CCTV and home alarm systems cannot get a software update whereas the proposed system can get software updates. From the developer point of view, this is significant. Because any bugs in the system can be rectified and the performance can be improved. The cost of existing system are too high, the CCTV requires rupees ten thousand to thirty thousand. The cost of installing a single raspberry pi camera is around rupees four thousand only, this could vary if the user opt a higher resolution camera.

\section{CONCLUSION}

In this paper we proposed a security system, which is able to monitor the home perimeter and notify the owner when a visitor intrudes. The system is able to notify the user instantly through email, SMS and even through a Whatsapp message. Unlike the existing CCTV systems, the software of the proposed system can be updated. It is an efficient and cost effective security solution for home security automation. The advantage of using this system is that, it is very user friendly and more importantly it is very simple to operate. In future the system could get updates for human face detection and thus eliminating unwanted motion detections

\section{ACKNOWLEDGMENTS}

We take this opportunity to convey our deep and sincere thanks to the Principal of MES college of Engineering Kuttippuram, Dr.V.H Abdul Salam and Head of the computer science Department Prof. Mredhula L. We also ex-tend deep gratitude to all the Professors of Computer Science Department of MES college of Engineering Kuttippuram for their valuable help and support. We express our sincere gratitude to all the students of department of Computer Science and our beloved family members who helped us with their timely suggestion and support. we also express our sincere thanks to all of our friends who helped us through out the successful completion of the work. All glory and honor be to the Almighty, who showered his abundant grace on us to make this work successful.

\section{REFERENCES}

[1] Raspberry Pi board - raspberry pi foundaton's official website http://www.raspberrypi.org/help/faqs/

[2] Gareth, Mitchell "The Raspberry Pi Single-Board" Engineering and technology 7.3 (2012)8.

[3] TOA Corporation, TOA Electronics, Inc. (USA) "Closed Circuit Television Systems Fundamentals Course", April 2005

[4] Alarm Systems "A Guide to Design, Management and Procurement", The Engineering Equipment and materials Users Association (EEMUA) publication No.191.

[5] Raspbian -the operating system used in this paper http://www.raspbian.org/Raspbianimages

[6] Gantt, Charles. "Raspberry Pi Camera Module Review and Tutorial Guide" TweakTown News. Tweak Town, 2 July 2013. Web. Oct. 2013 http://www.tweaktown.com/guides/5617/raspberry-picamera-module-review-and-tutorial-guide/index $4 . \mathrm{html}$

[7] Motion_Detection_Programming_Guide_V1.1 GM8126

[8] Python Sending Email Using SMTP. "Tutorials Point Simply Easy Learning." N.p., Web. Oct.

2013. http://www.tutorialspoint.com/python/python_sen ding_email.htm.

[9] Twilio - APIs for Text Messaging, VoIP \& Voice in the Cloud https://www.twilio.com/help

[10] Yowsup- unoficial whatsapp client for Linux https://www.github.com/tgalal/yowsup 